\title{
Link Residual-Time Estimation for VANET Cross-Layer Design
}

\author{
Nikoletta Sofra, Athanasios Gkelias and Kin K. Leung \\ Electrical and Electronic Engineering Department \\ Imperial College London, UK \\ \{nsofra,a.gkelias, kin.leung\}@imperial.ac.uk
}

\begin{abstract}
Traditional network design may underestimate the dependencies between different layers of the protocol stack and fail to exploit the direct coupling of physical-layer operations to the network topology. In the case of a highly dynamic network, as observed in vehicular communications, the network architecture needs to be able to optimally adapt to the changes brought on by nodes' mobility. In this paper we propose a crosslayer approach, where the received power metric, logged at the physical layer, can be used to produce estimates of the links' residual lifetime. Such information is crucial for the decision processes of higher layers, such as hand-off, scheduling and routing. The method comprises of utilizing a time series based on physical-layer measurements to estimate the current state of the link and the remaining time during which the link can be used for efficient communication. Shadowing, small scale fading and limited opportunities to sample the channel make the problem challenging. The proposed algorithms are tested against simulations which depict the mobile wireless channel realistically. Keywords-Vehicular Ad hoc networks, Link Lifetime, Empirical Mode Decomposition
\end{abstract}

\section{INTRODUCTION}

Vehicular Ad hoc Networks enable vehicle to vehicle and vehicle to roadside communication, having a wide range of technological applications. VANETs form a subset of Mobile Ad hoc Networks, but are distinguishable from MANETs in many ways, as they exhibit rapid topology changes, frequent fragmentation, relatively small network diameter and limited redundancy [1].

One of the biggest challenges in VANETs is the high mobility of the nodes, which results in frequent fragmentation and short-lived links. It has been traditionally assumed that layers will be providing certain services to the higher ones seamlessly. However, in the case of high instability, upper layer protocols need to be able to adapt to changes affecting the communication capability, and they can only be designed to do so via cross layer interactions. For example, the routing procedure is strongly linked to the topology of the underlying network, assuming that connectivity with the neighbouring nodes is dealt with by underlying layers. Prior knowledge of

The work reported in this paper forms part of the MESSAGE project. MESSAGE is a three-year research project which started in October 2006 and is funded jointly by the UK Engineering and Physical Sciences Research Council and the UK Department for Transport. The project also has the support of nineteen non-academic organizations from public sector transport operations, commercial equipment providers, systems integrators and technology suppliers. More information is available from the web site www.message-project.org. The views expressed in this paper are those of the authors and do not represent the view of the Department for Transport or any of the non-academic partners of the MESSAGE project. existing links' residual time, their future quality or notifications of imminent breakage of a link could assist the routing or scheduling decisions to great extent.

The use of link quality feedback to the routing protocol in terms of physical layer information has been considered in many instances. More specifically, [2] proposes using the smoothed SNR value observed on the links as a metric for the routing decision and as a trigger for hand-off initiation. Similarly, Signal Stability-Based Adaptive routing [3] classifies the links as strong or weak and also takes their temporal stability into account; preemptive routing [4] elaborates on the value of the threshold below which a new route search is initiated. The authors of [5] propose using the received signal strength changing rate to predict the Link Available Time, but do not report taking the disadvantageous propagation conditions under which VANETs are operating into account as their work focuses more on the routing layer. Moreover, they consider the range of a node to be a constant known value, which might not always be the case. In contrast, the communication capability of two nodes depends on the achieved BER, which is affected by conditions related to the environment (e.g. shadow fading). Various methods to predict or study the link, or even route lifetime based on location and node movement information have been proposed, an example being [6], which assumes a free-space propagation model. There also exist different approaches which target at analytically deriving the lifetime of a link based on mobility models, such as [7].

The contribution of this work lies in proposing a method that estimates the link quality in terms of residual lifetime, based solely on physical layer measurements (received power) and without incurring any extra overhead in the traffic of the network. The evaluation of the method explicitly considers the unfavorable conditions of a mobile wireless channel (outdoor environment, multiple scatterers, moving obstacles, Doppler effect). The latter, together with the fact that the received power metric is numerically available only when packets are sent make the problem very challenging. These issues have been already underlined in [8-9]. The work presented here extends the method in [8], producing a more accurate model and gives further insight on the method's performance by studying the accuracy of both models in the presence of shadow and Rician fading.

More specifically, each link is monitored by logging the arrival time and received power level of every packet (traffic, control or hello packets) received on the link, thus introducing 
no extra traffic overhead on the network; this time series is then used to produce estimates of the metric. Mobile wireless communication channels are highly affected by both small scale fading, caused by the relative movement of the nodes, as well as shadowing. Moreover, shadow fading appears as a time-dependent variable, as the node moves through areas with different characteristics. Since our goal is to capture the change of the mean received power that is caused by change in the physical distance between the nodes, these phenomena introduce noise in our time series.

In summary, the approach we are taking is to develop a model for the distance-dependent change in received power over time as two nodes move relative to one another. After eliminating the noise induced by small scale and shadow fading to the possible extent, we acquire estimates for the parameters of the model. This enables us to identify whether the received power level increases or decreases over time, produce estimates of the link quality in future time points via extrapolation and detect the link breakage point. We mainly focus on the last metric, as we find it to be the most demanding. Link residual time (LRT) is defined as the remaining amount of time during which the link will remain useful for data transmission; that is to say the average received power shall remain over a specified threshold. Given the parameters of the model dictating the time dependence of received power on the large scale, an estimation of the time point on which this will fall below a specified threshold can be acquired.

\section{SYSTEM MODELLING}

The wireless mobile channel is affected by both shadowing and multipath fading in addition to the large scale path loss caused by change in the relative distance. Considering multipath fading and shadowing as independent random processes [10] we can model the received power as in:

$$
P_{R}=P_{T}-P_{L_{0}}-10 n \log _{10}(d)+\phi_{R}+\phi_{S} \quad(d B),
$$

where $P_{T}$ is the transmitting power, $P_{L o}$ a constant that depends on the frequency, antenna gains and other system loss factors, $n$ the path loss exponent depending on the propagation environment and $d$ the physical separation distance between transmitter and receiver, while $\phi_{R}$ represents the variations caused by multipath fading and $\phi_{S}$ the changes on the level of the received power due to shadow fading. Both $\phi_{R}$ and $\phi_{S}$ are considered as noise in this model. In the following we are aiming to derive an expression capturing the dependence of received power on time, when the mobile node(s) maintain(s) a constant direction of movement.

We are considering the link between a stationary node and a node that is moving with a constant direction on the $x y$ plane. This scenario covers both the case of a mobile node communicating with a road access point, as well as the one of two mobile nodes, $A$ and $B$, moving without changing direction. In the latter case, we consider node $A$ stationary and the equivalent relative movement of node $B$ relative to node $A$. Let $s$ be the axis on which node $B$ is moving (relative to $A$ ), and $\theta$ the angle between the line that is formed by the two nodes and $s$ (see Figure 1). In addition, let $s_{0} \in s$ be the point on which the distance between the two nodes is minimized and $d_{\min }$ the minimum distance achieved.

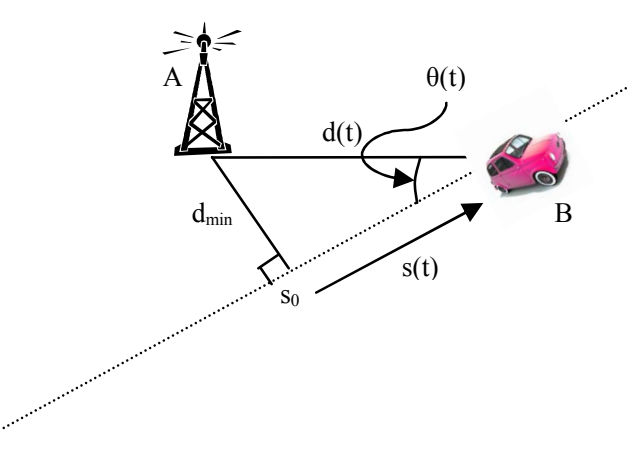

Figure 1. A mobile node moving in a constant direction relatively to a roadside access point.

In this context $s(t)$ represents the position of the mobile node on axis $s$, taking negative values before the node has reached $s_{0}$ and positive afterwards. This means that $s_{0} \equiv 0$ for the $s$ axis. In this scenario, we can distinguish two different phases on the nodes' relative movement; they are successively approaching and distancing each other. It follows that there are two different phases on the link quality variations on the large scale, namely improving and deteriorating. In our previous work we have underlined the importance of distinguishing between these phases, and how only measurements from the same phase should be used each time when making estimates. Whenever a change in phase is detected, all data belonging to a previous phase are discarded, and the beginning of time is set to coincide with the beginning of the corresponding phase.

If we assume that the speed of $B$ (or equivalently the relative speed of $\mathrm{B}$ with respect to $\mathrm{A}$ ), $u$, does not change significantly over a time interval defined as a phase (the phase detection process in section IV allows us to make this assumption), then

$$
\frac{\partial s(t)}{\partial t}=u
$$

From Figure 1 it is clear that the separation distance between the two nodes is given by

$$
d(t)=\sqrt{s(t)^{2}+d_{\min }^{2}} .
$$

Using the chain rule on (3) and substituting (2) we derive that

$$
\frac{\partial d(t)}{\partial t}=\bar{u} \cos (\theta(t)), \text { where } \begin{array}{ccc}
\bar{u}=-u & \text { for } & s(t) \leq 0 \\
\bar{u}=u & \text { for } & s(t)>0
\end{array}
$$

or simply that the rate of change of the separation distance with respect to time equals with the projection of the mobile node's velocity on the axis that is defined by the locations of the two nodes. We can now express the change of $d$ over time as

$$
d(t)=\bar{u} \int_{0}^{t} \cos \left(\theta\left(t^{\prime}\right)\right) d t^{\prime}+d_{t=0} .
$$

In the special case of the movement taking place along the axis defined by the two nodes, $\theta(t)=0$ for all $t$, and so we acquire a simplified version of (5):

$$
d(t)=\bar{u} t+d_{t=0}
$$


In fact, this simplification also works well for $d_{\min }$ being small enough compared to the nodes' range, as in that case $\cos (\theta(t)) \approx 1$ for the most part of the duration of the movement.

In order to acquire a more accurate expression, however, we need to quantify the dependence of $\theta$ with time:

$$
\theta(t)=\arctan \left(\frac{d_{\min }}{s(t)}\right)
$$

We consider that explicit knowledge of $d_{\min }$ would pose a very restrictive assumption on the problem. We have nevertheless observed that the rate with which $\theta(t)$ is changing is much bigger close to $s_{0}$, causing $\cos (\theta(t))$ to rapidly drop when the mobile is in the close area of $s_{0}$ and asymptotically approaching one as the distance increases. This means that for the distancing phase only (when $\theta(t)$ is decreasing with respect to time) we can coarsely approximate $\theta$ with the following equation:

$$
\cos (\theta(t)) \cong 1-\exp (c t), \quad c \leq 0,
$$

where $c$ is a non positive constant depending on $d_{\text {min }}$ and $u$, and equals zero for $d_{\text {min }}=0$. Putting (8) into (5) we have

$$
d(t)=\bar{u}\left(t+\frac{1-\exp (c t)}{c}\right)+d_{t=0},
$$

which is valid for the distancing phase only.

Based on the above analysis, we may derive two different models for the received power; putting (6) into (1) yields

$M_{1}: P_{R}(t)=a-10 n \log _{10}(b t+1)+\varepsilon$,

while (9) combined with (1) gives

$$
M_{2}: P_{R}(t)=a-10 n \log _{10}\left(b\left(t+\frac{1-\exp (c t)}{c}\right)+1\right)+\varepsilon \text {. }
$$

The second model is valid for the distancing phase only. This does not pose a serious constraint on acquiring LRT estimates as these are only produced when a declining trend in received power is observed.

In the above models $\mathrm{c}$ is the corrective constant used in (9),

$$
a=P_{R}\left(d_{t=0}\right) \quad \text { and } \quad b=\frac{\bar{u}}{d_{t=0}}
$$

\section{DENOISING}

As has already been mentioned, the samples of received energy are affected by multipath and shadow fading, which introduce noise in our data. It is therefore necessary to introduce a noise removal process prior to estimating the model parameters. For this reason, we employ a novel signal processing technique, Empirical Mode Decomposition (EMD) [11-12], suitable for non-stationary and non-periodically distributed time series.

Very briefly, EMD gradually dissects the time series into a case-dependent number of intrinsic mode functions (IMFs). The method includes the computation of the lower and the upper envelope of the signal by interpolating between local extrema. The local trend can then be identified as the mean of the envelopes, while the local detail is what remains. After a sifting process on the local detail the corresponding IMF is extracted and the procedure is iterated. IMFs go from finer to coarser temporal scale and thus from higher to lower frequencies. Noise and channel fading will naturally occupy the higher frequencies, while changes on the large scale will reside on the lower ones. We can diminish higher frequencies by only keeping the later extracted IMFs, thus achieving a smoothing of the original samples. We are using a criterion as in [15], which does not assume any knowledge of the noise level; we may choose which IMFs to keep based on the change on the energy of each IMF. The IMF of the smallest energy, be $\mathrm{IMF}_{\mathrm{j}}$, is considered the threshold between noise-dominated IMFs and signal-component dominated ones. We therefore discard all the IMFs derived before $\mathrm{IMF}_{\mathrm{j}}$.

\section{PHASE DETECTION}

A critical part of the method proposed is the capability to detect between the different phases of movement that have been defined in section III. As the second model (10b) is only valid for the distancing phase, we can not use the adaptive method described in [8], but follow a similar technique.

Robust regression is an iterative form of simple regression, where each observation is assigned a weight based on the residuals from the previous iteration. Upon receipt of a new packet of power $P_{n}$, robust regression is used to fit the denoised time series of received power linearly with time, as in $P_{r}(t)=\lambda_{n} t+\rho_{n}$. This is a rather crude approximation, nevertheless it is faster than performing non-linear model parameter estimation and it enables us to detect a change of phase: the residual (defined as the mean square error between the observed values and those arising from the regression model) $r_{n}$ is stored and compared with the residuals from previous steps $\left(r_{1}, r_{2, \ldots}, r_{n-1}\right)$ in order to detect a possible change of phase. In that case, all previous samples are discarded. The sign of $\lambda_{n}$ also serves link classification purposes.

\section{LINK RESIDUAL TIME ESTIMATION}

During a deteriorating phase, we wish to detect the residual time for which the link will remain useful for data transmission. After estimating the parameters of the two models (10) using non linear parameter estimation methods, we may acquire an estimate of LRT, by determining the time point when the received power is expected to drop below a specified threshold. When using the first model (10a) the residual time will be given by

$$
\operatorname{LR} T(t)=\frac{10^{\frac{\gamma-\hat{a}_{P}}{10 n}}-1}{\hat{b}_{P}}-t,
$$

where $\gamma$ is the threshold value of received power, in $\mathrm{dbm}$, for efficient communication. Alternatively, based on (10b) and the estimates of parameters $a, b$ and $c$ we can estimate LRT using a numerical method, in order to find the time point $t_{t h}$ at which the received power is expected to be equal to $\gamma$. This adds an additional level of complexity in using the second model but is justified on the grounds of improved accuracy.

As the link residual time is computed consecutive times during the phases when received energy is deteriorating, we introduce convergence criteria on whether to accept the estimated value. Naturally, the more samples available, the better estimates are acquired, and there is a trade-off between the mean accuracy of our estimations and the time before the first estimates are accepted. 


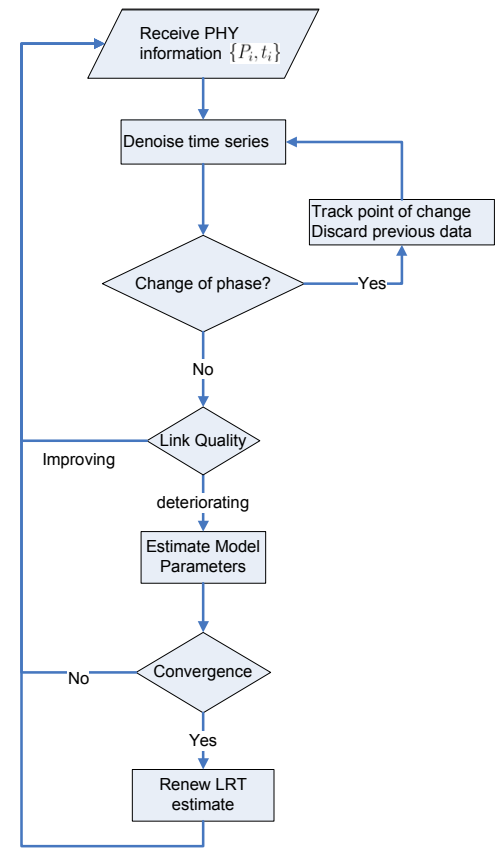

Figure 2. Flowchart of the proposed method.

The whole process of acquiring estimates for LRT is shown in Figure 2.

\section{Simulation Evaluation}

In order to assess the performance of the proposed algorithms a simulation platform has been implemented in Matlab. In these simulations the impact of the channel shadowing and the distance of the access point from the velocity vector on the efficiency and prediction error of our algorithm is evaluated based on the communication scenario depicted in Figure 1. As it has been discussed, this scenario can also cover the case of two mobile nodes whose directions are not changing for the duration of their movement. For the channel modeling we consider line of sight (LOS) communication with lognormal shadowing and Rician fading. The carrier frequency is $2.4 \mathrm{GHz}$ according to the IEEE $802.11 \mathrm{~g}$ standard, and the path loss exponent is set to $\mathrm{n}=3.5$. The speed of the mobile node is set to $15 \mathrm{~m} / \mathrm{sec}$ but is contaminated with additive white Gaussian noise $(\mathrm{SNR}=10)$, so that it varies for the whole duration of the movement, while the shadowing is assumed to have a correlation distance [16] of $5 \mathrm{~m}$. The threshold for efficient communication is considered to be $\gamma=-96 \mathrm{dbm}$, although we assume that packets of power down to $-100 \mathrm{dbm}$ can be detected by the node, and their power can be measured and logged.

We assume that the physical layer information is passed on to the algorithm every $T_{\text {agg }}$ which is set to $250 \mathrm{msec}$, in the form of the mean received power over all packets received on that link (if there exist any) during the corresponding period. Since $T_{\text {agg }}$ is in the order of some hundreds milliseconds the large scale fading does not change considerably for normal vehicular speed. In this way computational demand will be lower, as the algorithm needs only to be updated once, utilizing all the information acquired within the sampling period.

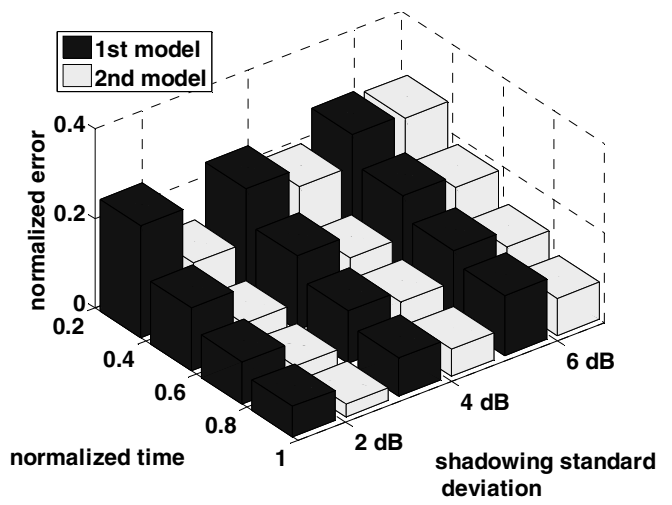

Figure 3. Effect of shadowing standard deviation on the normalized error per time interval.

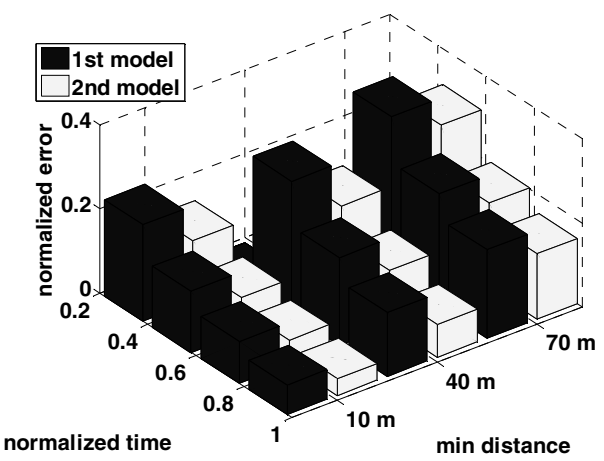

Figure 4. Effect of the minimum distance achieved on the normalized error per time interval

The implementation of Empirical Mode Decomposition used is as described in $[12,14]$. The non linear parameter estimation method used is a subspace trust region method, based on the interior-reflective Newton method [15].

The samples are distributed uniformly in time in such a way so that on average $20 \%$ of the time the channel is known, either by existing traffic on this link, or by eavesdropping on the packets the specific neighbor is sending to other destinations. A study of the effect of this parameter when using the first model with Rayleigh fading has been given in [8].

We examine the effect of different parameters on the accuracy and convergence of the LRT estimations, comparing the use of the two models (10). We define the normalized error as the absolute value of the ratio between the difference of the estimated minus the actual residual time, over the actual time from the point where the minimum distance between access point and mobile node achieved (reference point) to the point where the communication is lost $\left(T_{d}\right)$. Moreover, we define as normalized estimation time $\left(T_{e}\right)$ as the time that the estimation took place (measured from the reference point) over $T_{d}$. As the standard deviation of the error of consecutive estimations is quite high, we further quantize the normalized time into intervals, each of them lasting $20 \%$ of the deteriorating phase of the corresponding channel realisation so that we can demonstrate how the estimations improve as time passes and the number of available samples increases.

The behavior of the methods for different levels of shadowing, with the standard deviation of shadowing ranging 


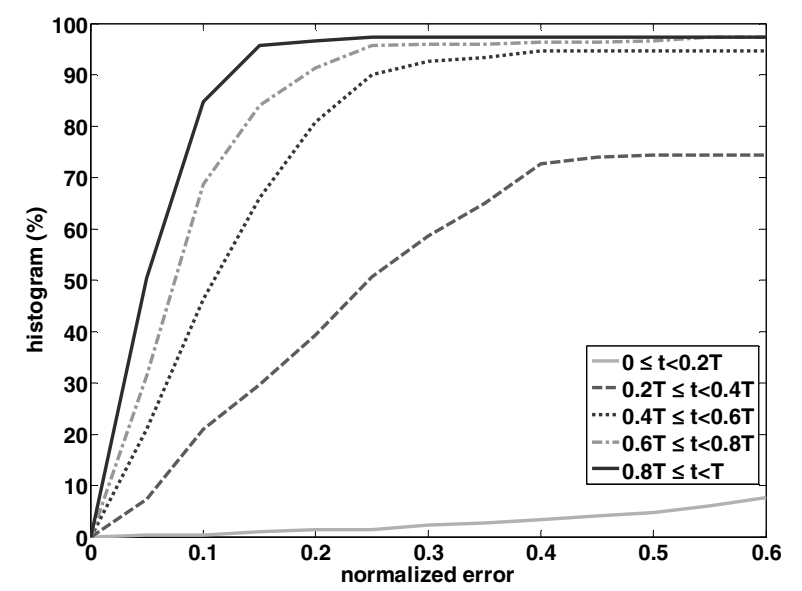

Figure 5. Empirical distribution function of the normalised error for each time interval.

from 2 to $6 \mathrm{dbm}$ is depicted in Figure 3. In total, 300 different time series for each value of deviation were simulated. The minimum distance was set to $d_{\min }=10 \mathrm{~m}$. As expected, the accuracy decreases with the shadowing deviation, as the data contain more noise. For example, the mean normalized error of all iterations during the last time interval in Figure 3 when using the $2^{\text {nd }}$ model is $2.83 \%, 6.05 \%$ and $8.3 \%$ for shadowing deviation of 2, 4 and $6 \mathrm{~dB}$ accordingly. The $1^{\text {st }}$ model had an accuracy of $6.82 \%, 8.58 \%$ and $13.42 \%$ in the same settings.

The effect of the minimum distance achieved is shown in Figure 4. Shadowing was simulated at $3 \mathrm{~dB}$, and 300 different channel realizations were tested for each value of $d_{m i n}$. As the minimum distance increases, the total duration of the movement decreases, as the mobile node will go out of range sooner. This in turn means that $T_{d}$ will be smaller, so for the same level of absolute accuracy, the normalized error will be bigger as the minimum distance increases. In all cases we plot the mean normalized error along the time interval of all the Monte Carlo iterations that have converged. If less than $50 \%$ of the iterations have not yet converged, the mean error does not appear in the plot. It follows that in Figure 4 fewer than $50 \%$ of the iterations had converged in the first two time intervals $\left(t<0.4 T_{d}\right)$ for $d_{\min }=40$ and $70 \mathrm{~m}$, so the mean error in that case was set to zero. We can see that the $2^{\text {nd }}$ model gives, again better accuracy than the $1^{\text {st }}$, especially in the cases of increased $d_{m i n}$. For example, when $d_{\min }=40 \mathrm{~m}$, the mean normalized error of the last time interval is $7.81 \%$ for the $2^{\text {nd }}$ model and $15.14 \%$ for the $1^{\text {st }}$.

Finally, Figure 5 demonstrates the impact of the time that estimation took place on the accuracy of the prediction, for the case of $d_{\min }=10 \mathrm{~m}$ with the standard deviation of shadowing at $4 \mathrm{~dB}$ when the $2^{\text {nd }}$ model is used. Very early predictions, i.e., $T_{e}<0.2 T_{d}$ are failing since the algorithms have not started to converge. However, for $T_{e}>0.4 T_{d}$, the proposed algorithm is able to provide accuracy higher than $80 \%$ for more than $80 \%$ of the time. While for $T_{e}>0.6 T_{d}$ accuracy higher than $80 \%$ can be achieved with probability higher than $90 \%$.

\section{CONCLUSION}

A method that utilizes the information available in the physical layer to estimate the residual life of communication links between mobile nodes when relatively sparse received power samples are available has been presented. Signal processing techniques and non-linear parameter estimation methods have been used. Simulation results demonstrated that the proposed algorithms can provide an accurate estimation of the residual life of wireless links well ahead before the communication failure occurs. This information is of paramount importance for the cross-layer design of efficient hand off, scheduling and routing algorithms for vehicular adhoc networks. Future work will include further evaluation of the aforementioned algorithms based on real measurements, possible adaptations to ensure compliance with $802.11 \mathrm{p}$ and integration of results on the design of upper layer protocols.

\section{REFERENCES}

[1] J.J. Blum, A. Eskandarian, L.J. Hoffman, "Challenges of intervehicle ad hoc networks," IEEE Transactions on Intelligent Transportation Systems, vol.5, no.4, pp. 347-351, Dec. 2004.

[2] T. Hsin-Mu, N. Wisitpongphan, O.K. Tonguz, "Link-quality aware ad hoc on-demand distance vector routing protocol," $1^{\text {st }}$ Int. Symp. on Wireless Pervasive Computing, p.6, 2006.

[3] R. Dube, C. D. Rais, W. Kuang-Yeh, and S. K. Tripathi, "Signal stability-based adaptive routing (SSA) for ad hoc mobile networks," IEEE Personal Commun., vol. 4, no. 1, pp. 36-45, 1997.

[4] T. Goff, N. B. Abu-Ghazaleh, D. S. Phatak, and R. Kahvecioglu. "Preemptive routing in Ad Hoc networks." In Proceedings of the 7th Annual International Conference on Mobile Computing and Networking (ACM MobiCom), Rome, 2001.

[5] R. Chang, S. Leu, "Long-lived path routing with received signal strength for ad hoc networks," 1st International Symposium on Wireless Pervasive Computing, Jan. 2006

[6] W. Su, S. Lee and M. Gerla, "Mobility prediction and routing in ad hoc wireless networks," Int. J. Network Management, vol. 11, no. 1, pp. 330, 2001.

[7] Y. Tseng, Y. Li, Y. Chang, "On route lifetime in multihop mobile ad hoc networks," IEEE Transactions on Mobile Computing, vol.2, no.4, pp. 366-376, Oct.-Dec. 2003.

[8] N. Sofra, K.K. Leung, "Link classification and residual time estimation through adaptive modeling for VANETs," IEEE Vehicular Technology Conference (VTC),Barcelona, Spain, April 2009.

[9] N. Sofra, K.K. Leung, "Estimation of Link Quality and Residual Time in Vehicular Ad Hoc Networks," IEEE Wireless Communications and Networking Conference(WCNC), Las Vegas, April 2008.

[10] G.L.Stueber, "Principles of Mobile Communication", Kluwer Academic Publishers, 1996.

[11] N. E. Huang, et al, "The empirical mode decomposition and the Hilbert spectrum for nonlinear and non-stationary time series analysis," Proc. of Mathematical, Physical and Engineering Sciences vol. 454, no. 1971, pp. 903-995, 1998.

[12] G.Rilling, P. Flandrin, and P. Gonçalves, "On Empirical Mode Decomposition and its Algorithms," IEEE Signal Processing Letters, vol.11, no 2, p.112, 2004.

[13] A.-O. Boudraa, J.-C.Cexus, "EMD-Based Signal Filtering," IEEE Transactions on Instrumentation and Measurement, vol.56, no.6, pp.2196-2202, Dec. 2007.

[14] "Empirical Mode Decomposition" [Online]. Available: http://perso.enslyon.fr/patrick.flandrin/emd.html [Acessed 28 Apr. 2009].

[15] T.F. Coleman and Y. Li, "An Interior, Trust Region Approach for Nonlinear Minimization Subject to Bounds," SIAM Journal on Optimization, Vol. 6, pp. 418-445, 1996.

[16] F. Perez Fontan, P. Mariño Espiñeira, "Modelling the Wireless Propagation Channel: A simulation approach with Matlab", John Wiley \& Sons Ltd 2008, p.67. 